\title{
Management of diabetic foot ulcer \& atherosclerosis using tibial transverse bone lengthening technique; Bangladesh experience
}

\begin{abstract}
Diabetic foot ulcers (DFU) are chronic skin ulcers associated with deep tissue destruction around the foot and ankle region with varying degrees of lower extremity vasculopathy and neuropathy. Diabetic foot ulcer is associated with a high rate of morbidity, disability, mortality and psycho-social cost.
\end{abstract}

Keywords: DFU, TTT, Osteotomy, ulcer, gangrene

Volume 13 Issue 5 - 202 I

\author{
Bari MM,' Choudhury Quayyum MKI, ${ }^{2}$ Islam \\ Shahidul, ${ }^{3}$ Ashraf Mohammad Tanvir, ${ }^{4}$ Bari AM \\ Shayan $\mathrm{R}^{5}$ \\ 'Prof. Ph.D, Chief Consultant, Bari-llizarov Orthopaedic Centre, \\ Visiting and Honored Prof., Russian llizarov Scientific Centre, \\ Kurgan \\ ${ }^{2}$ Prof. DG, BIRDEM (Bangladesh Institute of Research and \\ Rehabilitation in Diabetes, Endocrine and Metabolic Disorders), \\ Bangladesh \\ ${ }^{3}$ Dr. Md. Shahidul Islam, MD; FCPS, Prof., Bari-llizarov \\ Orthopaedic Centre, Bangladesh \\ ${ }^{4}$ Dr. Mohammad Tanvir Ashraf, D(Ortho), MS (Ortho), \\ Consultant, Orthopaedics, NITOR, Bangladesh \\ ${ }^{5}$ Dr.A.M. Shayan R. Bari, Medical officer, Bari-llizarov \\ Orthopaedic Centre, Bangladesh
}

Correspondence: Bari MM, Bari-llizarov Orthopaedic Centre, I/I, Suvastu Shirazi Square, Lalmatia Block E, Dhaka-1207, Bangladesh, Tel +880181921I595, Email bari.ilizarov3।@gmail.com

Received:September 10, 202I | Published: September 28, 2021

\section{Introduction}

Diabetic foot ulcer (DFU) is always associated with peripheral and automatic neuropathy that prevents with red swollen foot. We can easily identify clinically by seeing the presence of ulceration in the foot. The diabetic foot ulcer with deformity depends on some factors which includes osseous mal-alignment, trauma, metabolic abnormalities, and osteoporosis. The loss of vasomotor control permits vasodilatation that leads to increase peripheral blood flow, which increases arteriovenous shunting that causes bone resorption. Obesity and muscle atrophy do not allow the patient to bear weight. Sometimes osseous projections are the potential areas for diabetic foot ulcer. Osteomyelitis should be ruled out with ulcer. Infectious, ulcers and bone resorptions are always integrated. Dorsal and planter ulcers are very much common in diabetic foot. Medial column ulcers are associated with tarsometatarsal medial column collapse. Lateral column ulcers are more proximal to mid foot deformity that does not unite. Chronic ulcerations lead to deep infection and osteomyelitis. Surgical intervention and debridement are essential to remove all devitalized tissue. ${ }^{1,2}$

As an excellent treatment for diabetic foot ulcers our study at Bari-Ilizarov Orthopaedic Centre and BIHS (Bangladesh Institute of Health Science) showed that 50\% DFU are Wagner type 111 .

\section{Challenges of diabetic foot ulcer management}

i. The most common impaired chronic wound condition is in diabetic mellitus.

ii. $8 \%$ of population is estimated to suffer type 2 diabetes in 2035 . iii. $4 \%$ of diabetic patients develop foot ulcer each year $(15 \%$ during their life time)

iv. Diabetic foot refers to foot infection, ulcer and tissue destruction for peripheral vascular and nerve lesion.

v. Diabetic foot is one of the most serious and costly chronic complication of diabetes and severe cases can lead to amputation.

vi. About $85 \%$ of amputations are caused by foot ulcers.

vii. The relative risk of lower extremity disruption in diabetic patients is 40 times higher than non-diabetic patients.

viii. The 5 years mortality rate of DFU associated amputation is about $50 \%$

ix. Mortality rate of Wagner iv grade diabetic foot patients without surgery was as high as $54 \%$.

x. Significantly higher costs and social burdens.

xi. Novel and cost-effective management for diabetic foot ulcer is in burning need.

\section{Pathology of diabetic foot ulcers}

i. The vessels are calcified, that leads to disability.

ii. Overall, $45.6 \%$ of the subjects were found 'at risk' of DFU. This risk was higher among men (46.7\%) than women and among those who lived in rural areas (47.5\%) as compared with the urban population. 
iii. Primary care physicians and diabetic patients usually have a low degree of awareness of diabetic vascular lesions.

iv. Microvascular damage increases blood viscosity and blood flow disorder.

\section{Conventional treatments}

i. Blood glucose control.

ii. Blood pressure and lipid control.

iii. Nutrition supplement therapy for the patients who are suffering with kidney disease, malnutrition, and hyperproteinemia.

\section{During and after tibial transverse technique patient reports}

i. Relief of pain,

ii. The limb becomes warmth.

iii. Distraction osteogenesis increases capillary and vascular network.

\section{Biological mechanisms}

\section{A. Corticotomy}

i. llizarob corticotomy is a low energy osteotomy with the preservation of periosteum, endosteum and bone marrow.

ii. In choosing corticotomy level, anatomic, biomechanical and physiological factors must be considered.

\section{B. Law of tension stress}

Ilizarov's law of tension-stress promotes formation of

i. Neovascularization

ii. Increases collateral circulations in the affected limbs

\section{Tibial transverse technique}

Kurgan evidence suggests that Tibial Transverse Technique may mobilize stem cells and helps in formation of mesenchymal stem cells towards damaged tissues.

\section{Wagner DFU Classification}

The Wagner diabetic foot ulcer classification system assesses ulcer depth and the presence of osteomyelitis or gangrene by using the following grades:

i. Grade 0 - Intact Skin

ii. Grade 1-Superficial ulcer of skin or subcutaneous tissue

iii. Grade 11 - Ulcers extend into tendon, bone, or capsule

iv. Grade 11l- Deep ulcer with osteomyelitis, or abscess

v. Grade lV - Partial foot gangrene

vi. Grade V - Whole foot gangrene

\section{Principles of debridement for diabetic foot ulcers:}

i. Necrotic tissue must be removed.

ii. Treatment of infected lumens: Debridement should be done and we should leave the wound open.
Protocols for tibial transverse technique surgery - Basic requirements

We must have indications and contraindications for TTT.

\section{Indications:}

a. Chronic diabetic foot ulcer with atherosclerosis.

b. Inclusion criteria are:

- Diabetic foot with Wagner III and IV.

- The diabetic foot which received debridement, dressing change should go for TTT therapy.

- DFU combined with Wagner type II, III, or IV

- Patient interested to do the surgery.

\section{Contraindications}

Mentally ill patient who cannot assist the orthopaedic surgeon during treatment.

\section{Surgical procedure}

A small 5 incisions were made at the anterolateral part of the tibia. The distance between two incisions are $2 \mathrm{~cm}$. Through each 5 incisions a $5 \mathrm{~mm}$ osteotome is used to do the osteotomy so that we can osteotomize the incised tibia in a colinear fashion. In the proximal incision we should cut the bone $2 \mathrm{~cm}$ transversely. After that below each incision's osteotomy $1.8 \mathrm{~mm} 5$ olive wires were introduced to connect with lateral plate via slotted bars. The tibial section has been moved approx. $1 \mathrm{~mm} /$ day for 3 weeks. The clinical status improved within a few weeks. Cure of trophic ulcers and no more pain. Arteriogram on the 2nd months improved circulatory result. ${ }^{3-7}$

The diabetic foot ulcer under the great toe and little toe are seen in most of the cases. Pre surgery color duplex confirmed the extrusive occlusion of anterior tibia, posterior tibia, and anteria dorsalis pedis. After surgery with 5 days of interval we started distraction of the osteotomised bone with the plate. Tibial section has been moved approximately $1 \mathrm{~mm}$ per day for 3 weeks. The clinical condition improved in 3 weeks and cure of diabetic foot ulcer were seen.

A. Diagrammatic Scheme.

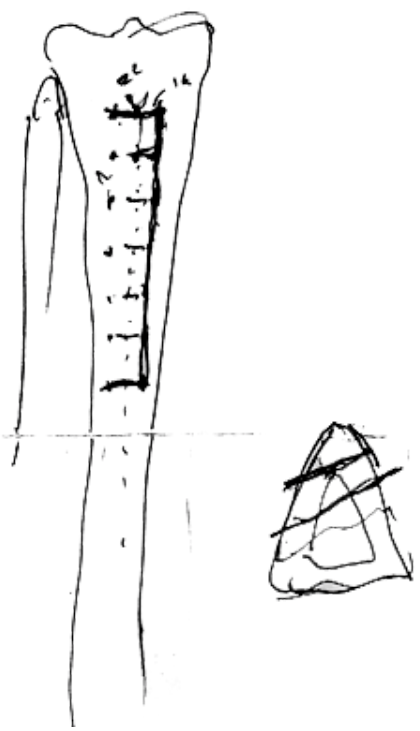


B. Tibial Transverse Technique and bone regeneration.

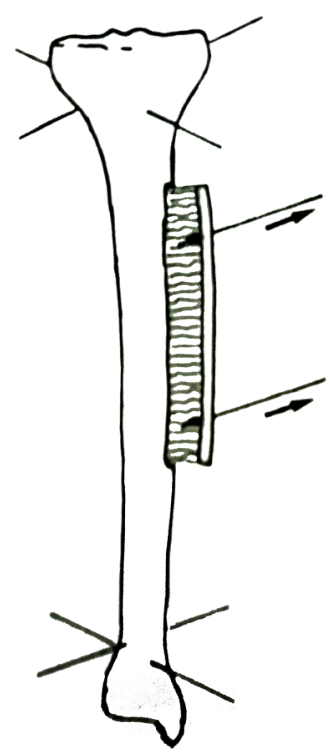

C. Distraction angiogenesis.

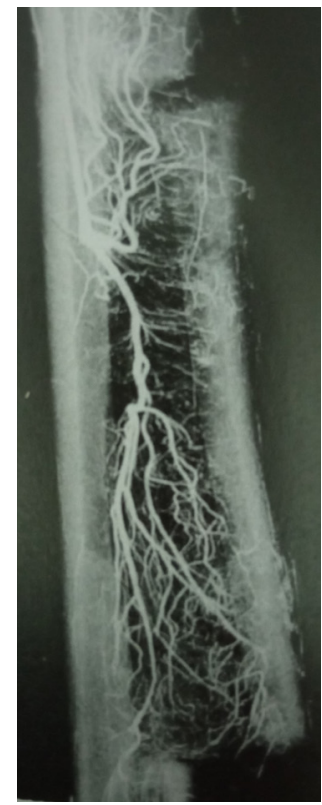

\section{Impact of neuropathy}

i. Signs and symptoms may be minimal

ii. Nevertheless, pathology proceeds rapidly

iii. End stage of tissue death is quickly reached

iv. Window of opportunity is very limited

v. Immune system and nervous system are deficient so body is unaware of bacterial invasion.

\section{Postsurgical management}

- Antibiotics should be used for 3-7 days

- Second postoperative dressing should be changed.

- To prevent limb oedema the limb should be raised up.

- Postoperative exercise is absolutely mandatory.

\section{Recent advances (Diabetic foot ulcers)}

Ilizarov discovered distraction histoneogenesis for limb regeneration. The tension-stress law could activate and enhance the regenerative potentials of living tissues, leading to growth or regeneration of muscles, fascia, blood vessels, and nerves simultaneously.

Clinical outcome of 58 cases of diabetic foot ulcers following Tibial Transverse Technique (TTT), bone lengthening

\begin{tabular}{ll}
\hline Total Cases & $\mathbf{5 8}$ \\
\hline Male & 46 \\
Female & 12 \\
Age (Years) & $35-72$ \\
Diabetes duration & $1-25$ \\
Foot Ulcer & \\
Duration & 3 days- I0 years \\
Wagner Grading & \\
Grade II & 25 \\
Grade III & 26 \\
Grade IV & 06 \\
Grade V & 01 \\
\hline
\end{tabular}

\section{Discussion}

In 2020, our study reported that 290 million adults worldwide had diabetes and it will rise to 450 million by the year 2035 . This big demographic shift shows the prevalence of diabetes chronic complications in the foot. If we continue the conservative therapy the foot ulcers do not cure, surgical debridement, dressings, strict glycemic control and intravenous antibiotic therapy may eradicate infection but foot problems remain life threatening to the patient, that's why we need to do distraction angiogenesis to increase microcirculation in the limb.

The Tibial Transverse Technique is based on Ilizarov's "law of tension-stress" for limb regeneration and functional reconstruction. The principle is slow and steady traction on a living tissue creates a stress and which is metabolically activated and which stimulates histoneogenesis". It promotes cell division and differentiation of adult stem cells like that in the fetal tissues development and lead to tissue regeneration. ${ }^{1-4}$

\section{Conclusion}

a. Tibial transverse technique for DFU (Wagner III and above) have successfully treated more than 58 cases. We used tibial transverse technique along with debridement and dressing change. ${ }^{1}$

b. Tibial transverse technique can significantly improve diabetic ulcer healing, increase the limb salvage rate and reduce the diabetic foot ulcer recurrence rate.

c. Tibial transverse technique was not $100 \%$ effective for severe cases of DFU.

d. The tibial transverse surgery should be performed with a minimally invasive fashion through small skin incisions, meticulous corticotomy, purposely-designed Ilizarov fixator, 
to ensure standard surgical procedure and postoperative management. ${ }^{1,7}$ e. We must follow the principles of surgical procedure. Pre and post-operative management which can help in success rate for tibial transverse surgery in severe diabetic foot ulcer treatment.

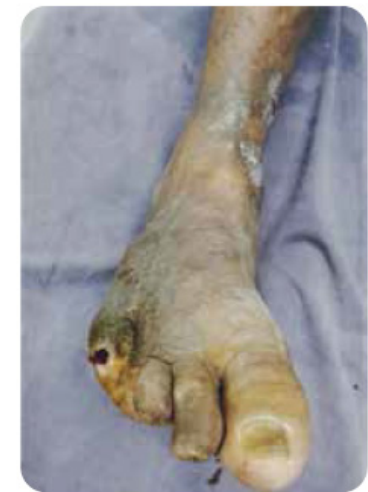

(1)

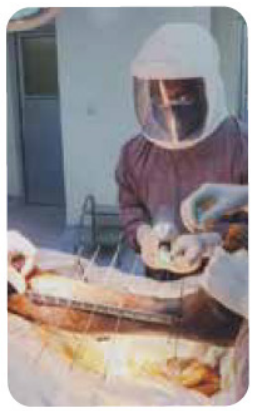

(4)

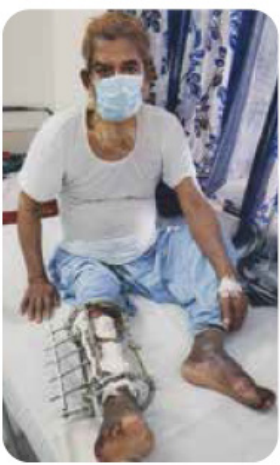

8

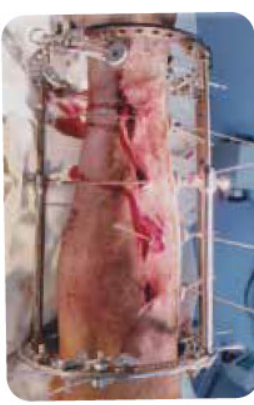

5

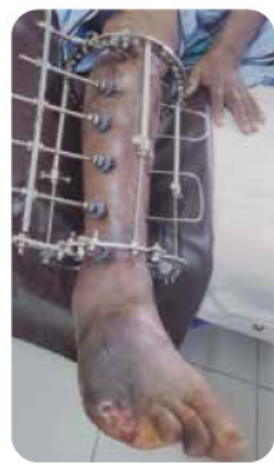

9

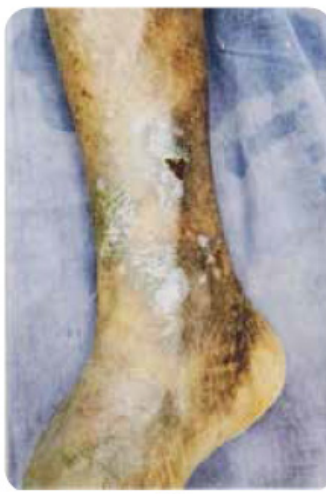

2

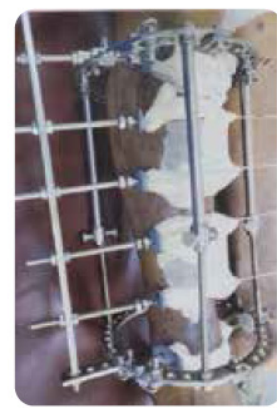

6

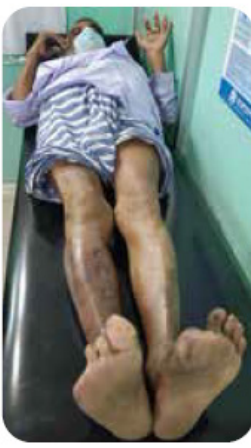

10

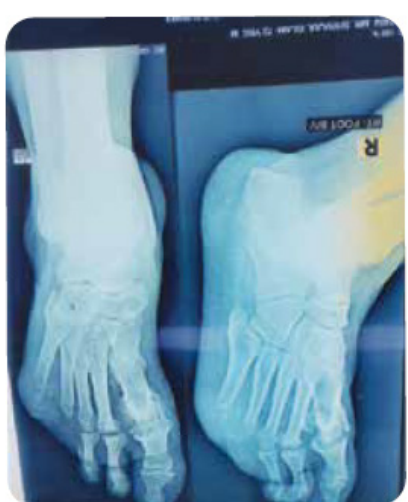

(3)

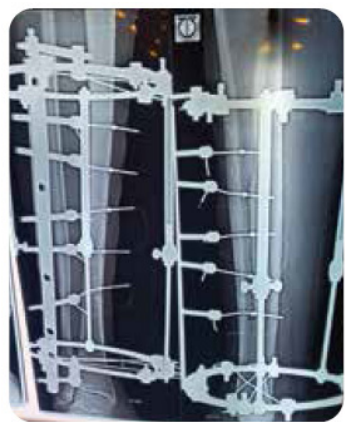

7

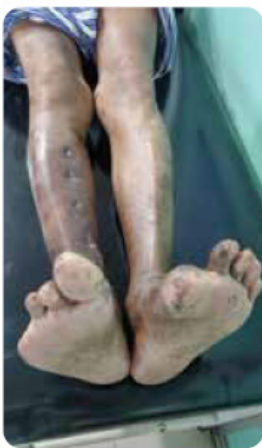

11

Figure I 72 years old man, Diabetic Foot Ulcer with atherosclerosis.

(I) Diabetic foot of right little toe ulcer with dry skin.

(2) Dry skin of lower tibia and ankle.

(3) Radiograph of right foot with resorption of right 5 th and 4th phalanges.

(4) In OR, multiple incisions and introducing the olive wires.

(5) In OR, long plate is fixing with the olive wires.

(6) View of Ilizarov fixation in the diabetic leg to increase vascularity.

(7) Radiograph of right foot with llizarov fixation, widening of tibia is seen

(8) After 14 days of Ilizarov fixation in the right leg, little toe ulcer is healed.

(9) View of the leg with llizarov fixation, skin color changed and ulcer is healed after 2 I days.

$(\mathrm{I} 0, \mathrm{I} \mathrm{I})$ Clinical appearance of the patient and right leg without ulcer.

Citation: Bari MM, Quayyum CMKI, Shahidul I, et al. Management of diabetic foot ulcer \& atherosclerosis using tibial transverse bone lengthening technique; Bangladesh experience. MOJ Orthop Rheumatol. 202I;I3(5):99-104. DOI: 10.15406/mojor.2021.13.00557 


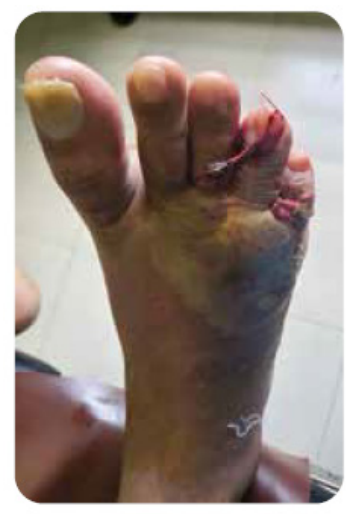

(1)

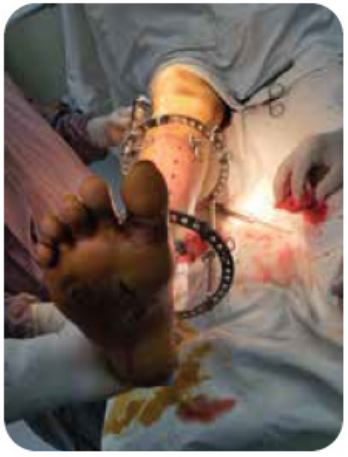

5

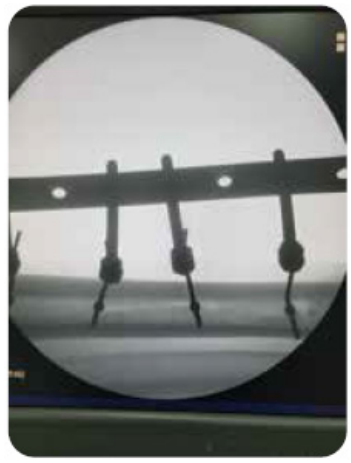

9

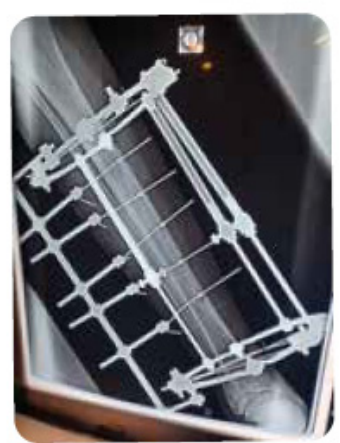

(13)

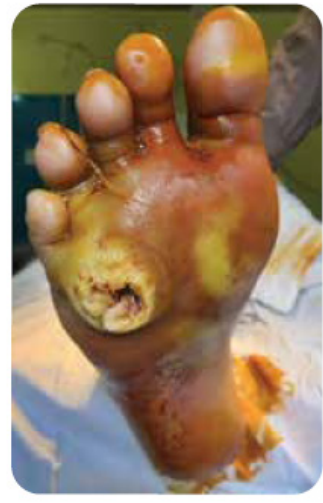

2

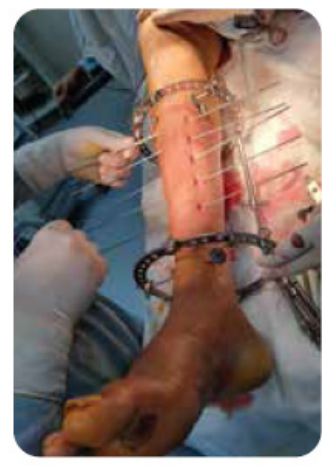

6

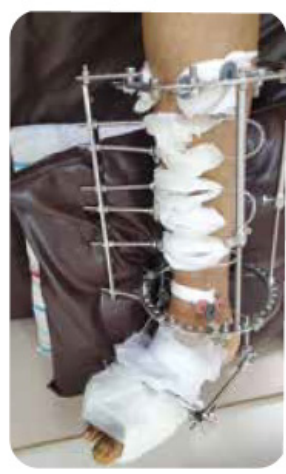

(10)

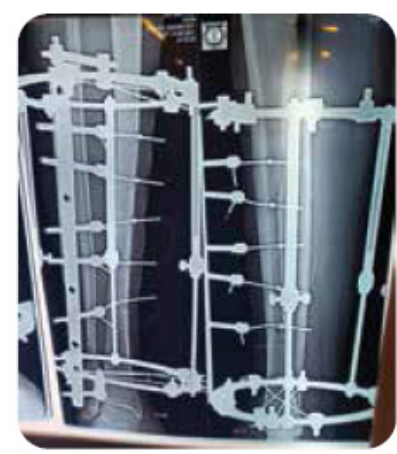

$(14$

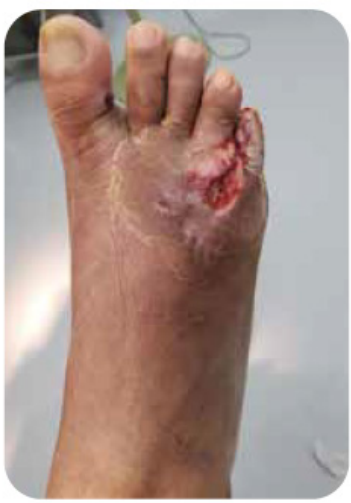

3

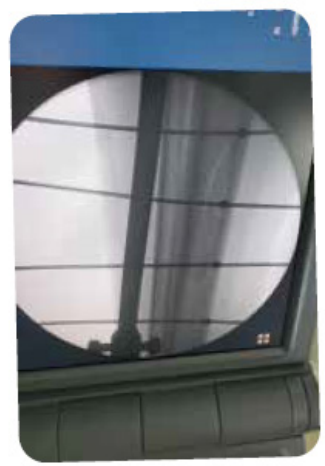

7

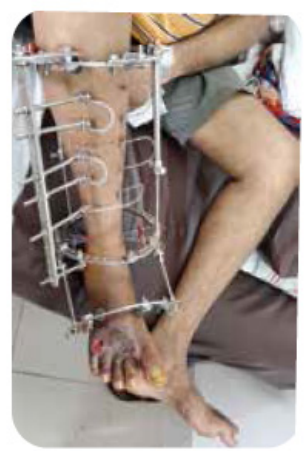

(11)

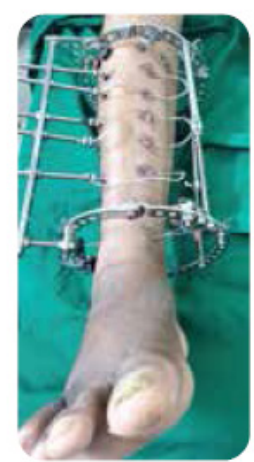

15

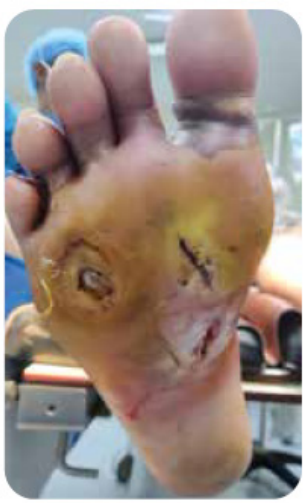

(4)

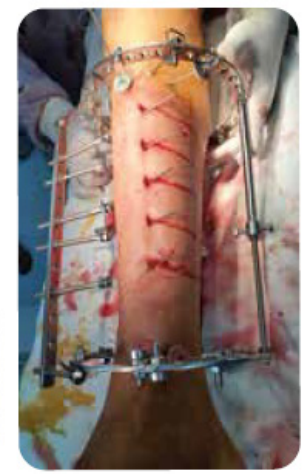

8

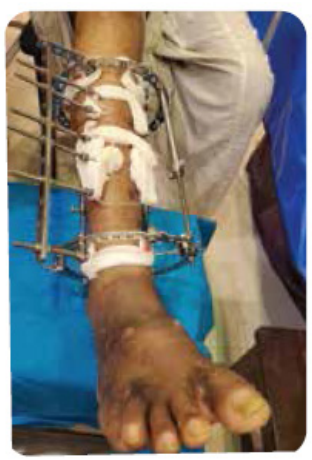

(12)

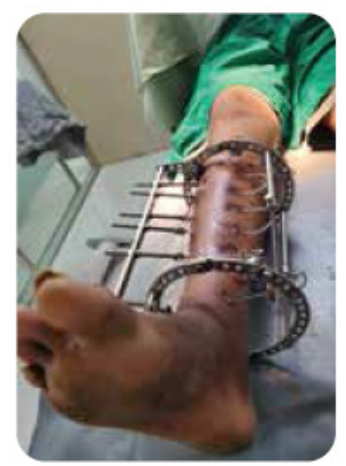

16 


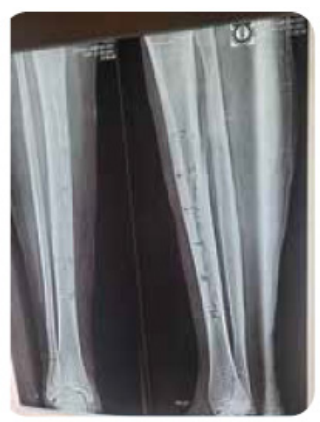

17

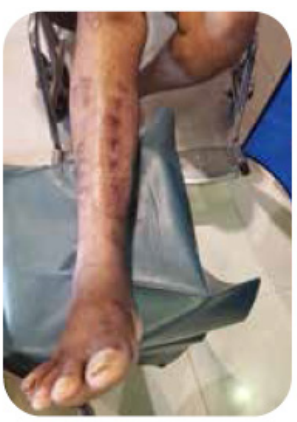

18

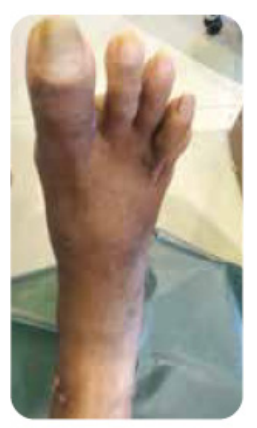

19

Figure 233 years old man, Diabetic Foot Ulcer with vasculopathy and neuropathy.

(I) Diabetic Foot Ulcer of dorsum of right foot, Wagner type III.

(2) Same patient, sole of the foot and dorsum of the great toe.

$(3,4)$ Dorsum and sole of the foot after debridement.

(5) In OR, multiple incisions seen in the tibia.

(6) In OR, placement of olive wires.

(7) Radiograph of right foot, placement of olive wires.

(8) Olive wires were connected through slotted bars and plate.

(9) Radiograph of distraction time.

(I0) View of placement of llizarov apparatus for TTT (Tibial Transverse Technique).

(II) 14 days after the TTT surgery.

(I2) After 2 I days, ulcer is healed.

$(13,14)$ Radiograph of right tibia with llizarov fixation, ulcer is healed (AP and Lateral view).

(I5) Before dismounting llizarov fixation, healed dorsum of the foot.

(16) Sole of the foot healed, before dismounting Ilizarov fixation.

(I7) Radiograph after removal of llizarov apparatus.

(I8-20) Final follow-up after 2 months without any ulcer.

\section{Acknowledgments}

None.

\section{Conflicts of interest}

The authors declare no conflicts of interest.

\section{References}

1. Ilizarov GA. The tension stress effect on the genesis and growth of tissues. Part I. The influence of stability of fixation and soft-tissue preservation. Clin Orthop. 1989; 238:249-281.

2. Ilizarov GA. The tension-stress effect on the genesis and growth of tissues: Part II. The influence of the rate and frequency of distraction. Clin Orthop. 1989;239:263-285.
3. Bari MM. A color atlas of limb lengthening, surgical reconstruction and deformity correction by Ilizarov technique. 2013;360-365

4. Ilizarov GA. Transosseous Osteosynthesis theoretical and clinical aspects of the regeneration and growth of tissue. Springer-Verlag Berlin Heidelberg. Germany. 1992. pp. 172

5. Jiang Y, Ran X, Jia L, et al. Epidemiology of type 2 diabetic foot problems and predictive factors for amputation in China. Int J Low Extrem Wounds. 2015;14(1):19-27.

6. Yang Y, Lin S, Wang B, et al. Stem cell therapy for enhancement of bone consolidation in distraction osteogenesis: a contemporary review of experimental studies. Bone Jt Res. 2017;6(6):385-390.

7. Qu L, Wang A, Tang F. Lateral tibial cortex transport surgery for vascular regeneration in the treatment of thromboembolic vasculitis. Chin Med J. 2001;81(10):622-624. 